\title{
Analysis of Heat Transfer for Solar Air Heater - A Perspective View
}

\author{
Shashi Shukla1, Prof. H. S. Sahu² \\ 1PG Scholar, ${ }^{2}$ Head of Department \\ 1,2Department of Mechanical Engineering, MIT, Bhopal, Madhya Pradesh, India
}

\begin{abstract}
Solar air heater is one of the most valuable heat sources of energy with variety of applications such as industrial process heating, space heating, drying of fruits and vegetables, etc. It is renewable as well as pollution free for space heating and when it is used in commercial buildings or industries could be very expensive. The solar air heating is a process in which the radiant energy emitted from the sun is captured on an absorber plate and is used for space heating. Improvement in thermal performance of a solar air heater can be done by enhancing the heat transfer. Providing artificial roughness on a heat transferring surface is one of the methods for effective enhancement of heat transfer to fluid flow, the other is to introduce the copper wire mesh screen matrix and by passing the fluid through it. In this paper, reviews of heat transfer techniques with the use of Artificial Roughness Geometry and wire mesh in order to improve performance and the effect of various parameters of porous media like pitch, number of layers, bed depth, porosity, thermal conductivity, pitch to wire diameter ratio have been discussed.
\end{abstract}

Keywords: Solar air heater, Copper mesh wire screen matrix, packed bed, Thermal efficiency

\section{INTRODUCTION}

Energy is available in many forms and plays an important role in worldwide economic industrialization and growth. The growth of world population and rising industrialization need large amount of energy. Among many renewable energy sources, solar energy is one of the huge energy sources for meeting this demand. The easiest way to utilize solar energy for heating applications is to convert it into thermal energy by using solar collectors. Solar air heater is a simple device that heats air by utilizing solar energy from the sun. Its applications involve drying of agricultural products, such as seeds, fruits, vegetables and space heating. Flat-plate solar collectors are extensively used in low temperature energy technology and attracted the attention of a large number of investigators. Several designs of solar air heaters have been developed over the years in order to improve their performance. Several methods have been used by various investigators to increase efficiency by using artificial roughness on the absorber plate, using fins and by using wire mesh. Drying and preservation of agricultural products have been one of the oldest uses of solar energy. The traditional method still widely used throughout the world, is open sun drying where diverse crops such as fruits, vegetable, tobacco, etc. are used and spread on the ground and turned regularly until sufficiently dried. Drying means preservation of food, fruits and vegetables for long time with good quality. Drying is a complicated process involving simultaneous heat and mass transfer. The required amount of energy to dry a particular product depends on many factors, such as initial moisture content, final moisture content, drying air temperature, relative humidity and velocity. Various mathematical models describing the drying behaviour of different food materials have been proposed to optimize the drying process and design efficient dryers. Modelling is advantageous because full scale experimentation of different products and configurations of drying system is very time consuming and costly. In order to improve the quality, the traditional natural sun drying must be replaced by modern drying methods. Drying characteristics of specific products should be determined to improve the quality.

\section{METHODS OF SOLAR DRYING TECHNOLOGIES} A. Direct Solar Drying

Direct solar drying is the conventional way of drying the products. In this method the products are directly exposed to the solar radiation and reduce the moisture content to atmospheric air. The air movement is due to density difference. It is broadly classified into two categories:

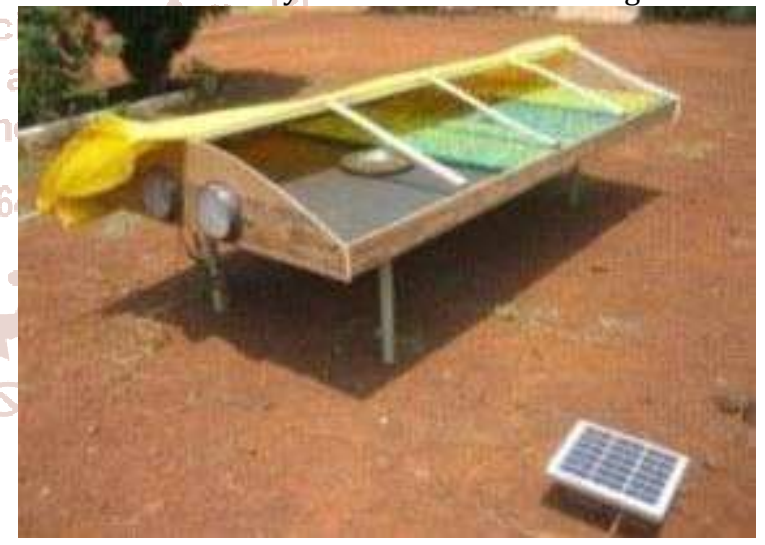

Figure. 1: Open Air (Direct) Solar Dryer.

Through a transparent cover which protects partly the foodstuff from rain and other natural phenomena i.e. a passive solar drying method. (Fig.2)

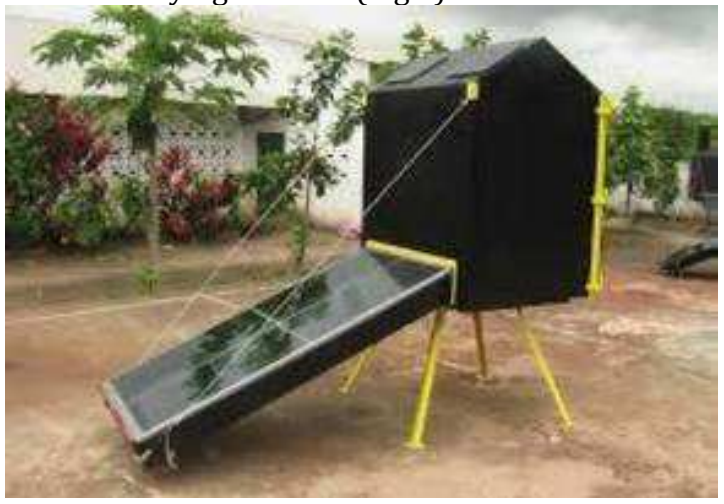

Fig. 2: Transparent Glass Covered Direct Solar Dryer. 
This technique involves a thin layer of product spread over large space exposed for a long time to solar radiation until the product will dry to a required level. The surface floor made from the concrete or particular area of soil is making applicable for Outdoor direct sun drying. This type of drying method is useful for grains. Material is laid over outdoor floor for a long time, usually 10- 30 days.

\section{B. Indirect Solar Drying}

Indirect solar drying or convective solar drying is the new technique of product drying. It is very efficient method than the direct type of solar drying. In this method the atmospheric air is heated in flat plate collector or concentrated type solar collector. The heating process is either passive or active. This hot air then flow in the cabin where products are stored. Therefore moisture from the product may lost by convection and diffusion. This method of drying is used to avoid direct exposing to the solar radiation. This method mainly reduces the disadvantages of direct solar drying.

\section{DEVELOPMENT \& CONCEPT OF ARTIFICIAL ROUGHNESS IN SOLAR AIR HEATER}

Artificial roughness provided under the absorber surface of solar air heater duct is one of the effective means to breakdown the laminar sub-layer and enhance heat transfer coefficient. Artificial roughness helps in creating favourable conditions for efficient heat transfer with minimal increase in friction factor by: disrupting laminar sub layer developed in vicinity of heat transferring surface; increasing turbulence intensity; increasing heat transferring surface area; generating vortex and / or secondary flows.

\section{A. Roughness in the form of ribs / wires}

Raj Kumar, Ranchan Chauhan, Muneesh Sethi[10] carried out experimental investigation to study heat transfer and friction characteristics of solar air heater artificially roughened with discrete $\mathrm{v}$-down ribs on one broad wall as shown in figure (3).

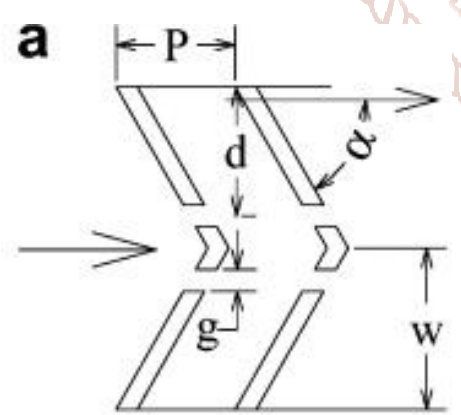

Figure 3: Discrete V-down arrangement

From various authors it was concluded that there was about 3.04 and 3.11 times enhancement in heat transfer and friction factor compared to the smooth duct. Also the correlations for $\mathrm{Nu}$ and $\mathrm{f}$ were determined and investigated the effect due to V-shaped ribs with gap on heat transfer and friction characteristics of solar air heater duct. It was concluded that there was about 6.32 and 6.12 times enhancement in heat transfer and friction factor compared with smooth duct. Also the correlations for Nusselt number and friction factor were developed by the authors within the range of investigated parameters.

\section{B. Roughness in the form of Dimples / Protrusions}

Raj Kumar, Ranchan Chauhan, Muneesh Sethi[10],developed correlations for Nusselt number and friction factor for solar air heater duct artificially roughened with protrusions on one broad wall. It was concluded that there was 2.89 and 2.93times enhancement in heat transfer and friction factor. The schematic diagram of roughened absorber plate is given in Fig. 4. Experimental investigation carried out by different authors with protruded roughness geometry also confirms 3.8 and 2.2 times enhancement in heat transfer and friction factor, carried out investigations over dimpled roughness geometry arranged in arc shape and concluded that maximum enhancement in heat transfer takes place at $\mathrm{p} / \mathrm{e}$ of 10 and angle of attack $60^{\circ}$. Thus it can be concluded that protrusions / dimples in different shapes and orientations helps in heat transfer enhancement with minimal increase in friction factor.

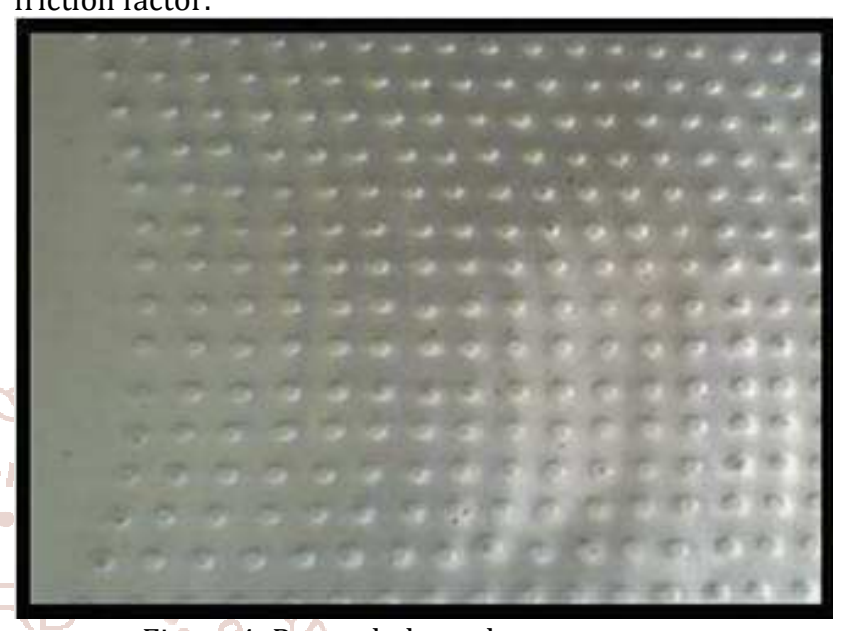

Figure 4: Protruded roughness geometry

\section{Roughness in the form of Baffles}

An experimental investigation was carried out by Raj Kumar, Ranchan Chauhan and Muneesh Sethi [10], to assess forced convection heat transfer and friction behavior for air flow in a channel fitted with multiple, $60^{\circ} \mathrm{V}$ shaped baffles. Considerableenhancement in heat transfer was obtained with baffles operating under e/ $\mathrm{H}$ of $0.10,0.20,0.30$; PR equal to 1 , 2 and 3 and the transverse pitch of $\mathrm{V}$-baffle was $\backslash$ set at $2 \mathrm{H}$. The effect of solid Z-shaped baffles on heat transfer augmentation fitted at $45^{\circ}$ in phase and out phase was investigated experimentally by authors aligned in zigzag fashion on the isothermally fluxed top wall. The effect of baffle height and pitch spacing was examined to arrive at optimal values for maximum enhancement in thermal performance. Baffles with different orientations help in increasing heat transfer. However due to recirculation of the flow, hot zone gets developed just behind the baffles which deteriorate the heat transfer from these zones. This problem has been identified by the researchers and attempted to provide perforations in the baffles which allow a part of flow to pass through these perforations and mix with the main flow which creates higher level of mixing and creates turbulence which enhance the rate of heat transfer. Authors experimentally studied the effect of such perforated baffles as shown in Fig. 5 and obtained an enhancement of about 79 $169 \%$ in Nusselt number than that of smooth duct.

\section{Fully perforated baffles and half perforated baffles with two rows of holes and single row of holes respectively}

An experimental investigation was carried out by Raj Kumar, Ranchan Chauhan and Muneesh Sethi [10], the use of baffles half or fully perforated exhibits higher rate of heat transfer than solid baffles. Thus experimental investigations should be 
carried out using perforated baffles inline or staggered and with different orientations to determine the best suited geometry and optimization of the related working and operating parameters may be boost the system towards commercialization.
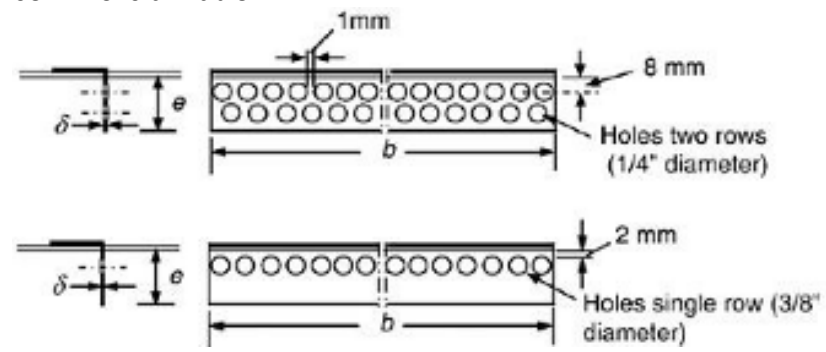

Figure 5: Fully perforated baffles and half perforated baffles with two and single row of holes respectively

\section{E. W-Shaped Artificial Roughness Ribs}

Manish Kumar Tated, Dinkar Prasad Singh and Sudhanshu Dogra [3] investigated that GI sheet of $0.8 \mathrm{~mm}$ thickness is used as absorber plate. Aluminum wire of $2 \mathrm{~mm}$ diameter is used as roughness element and this wire is attached on both side of plate. The absorber plate is shown in "Figure.6". An experimental investigation has been carried out to show the effect of relative roughness pitch on heat transfer and friction factor of double pass solar air heater having $\mathrm{W}$-shaped artificial roughness on both sides of absorber plate. On the basis of experimental investigation it is found that by providing artificial roughness on both sides of absorber plate both heat transfer and friction factor enhanced. It has been seen that Nusselt number increases continuously with increase in Reynolds number whereas friction factor continuously decreases with increase in Reynolds number. At relative roughness pitch $(\mathrm{p} / \mathrm{e})$ of 10 maximum heat transfer and friction factor occurs. Nusselt number enhanced 1.4 times as compare to smooth duct. By using this concept the highly efficient solar air heater can be design.

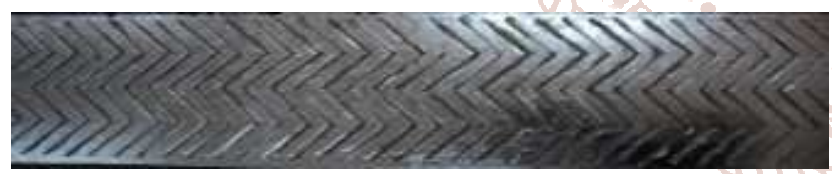

Figure6. Pictorial view of absorber plate

\section{F. Transverse V- Porous Ribs}

Santosh Vyas and Dr. Sunil Punjabi [11] investigated that mesh when made of aluminium wires of $0.5 \mathrm{~mm}$ diameter and having square openings of $2 \mathrm{~mm} \times 2 \mathrm{~mm}$, while thickness is $0.95 \mathrm{~mm}$. Width of each rib is $8 \mathrm{~cm}$. A gap of $22 \mathrm{~cm}$ is provided between first rib and second rib from inlet side for uninterrupted projection of laser beam and to accommodate thermal sensors. Keeping other conditions same as discussed in section 4.1, an air mass flow rate of $1.03 \mathrm{gm} / \mathrm{sec}$ is obtained with a temperature rise of $38^{\circ} \mathrm{C}$, which is $6^{\circ} \mathrm{C}$ higher than previous one. Overall thermal efficiency thus calculated is found to be $17.24 \%$ (a percentage increment of $15.62 \%$ ) with this modification in the design.

\section{G. Inclined V-Porous Ribs}

Santosh Vyas and Dr. Sunil Punjabi [11] the ribs are positioned inclined to each other at right angle. This modification is adapted so as to increase the portion of air flowing across the mesh and in turn air can absorb more amount of heat from absorbing surfaces. In the present experiments, testing has been conducted under natural convection keeping other conditions same as discussed in sections 4.1 and 4.2. Overall thermal efficiency obtained was $20.04 \%$ with temperature rise of $45^{\circ} \mathrm{C}$ and air mass flow rate of $1.011 \mathrm{gm} / \mathrm{sec}$. Thus a change in design has contributed in percentage rise of $34.4 \%$ in overall thermal efficiency with respect to basic design (first model) and of $16.24 \%$ as compared to transverse $\mathrm{V}$-porous rib design (second model).

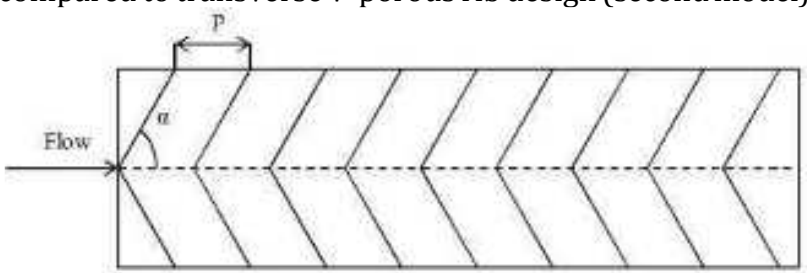

Figure 9. Solar air heater with inclined V-porous ribs

\section{H. Wire meshed}

K. Rajarajeswari, A. Sreekumar [4] investigated that many researchers have designed and studied matrix solar air heaters with different mesh parameters. Single and double pass solar air heater with wire mesh as packing bed. The collector design consists of 10 layers of steel wire mesh as absorber. The porosity of the packing was above 0.85 . System was studied for different mass flow rates. Other researched that $2 \mathrm{~mm}$ thick aluminium sheet as absorber plate and several layers of wire mesh screens packed one above the other. Thermal and thermo-hydraulic performance of the system for parallel and counter flow was studied both theoretically and experimentally with varying bed porosities and mass flow rates. The study reported that thermal efficiency of packed bed heater decreased as the porosity increased. In their study, twelve sheets of steel wire mesh arranged in 6 layers with 3, 3, 2, 2, 1, 1 sheets from bottom to top respectively. The diameter of the wire used was $0.02 \mathrm{~cm}$ with $0.18 \mathrm{~cm} \times 0.18 \mathrm{~cm}$ cross sectional opening of the layers. Results show that thermal efficiency increases with increasing mass flow rate between $0.0121 \mathrm{~kg} / \mathrm{s}$ and 0.042 $\mathrm{kg} / \mathrm{s}$ and maximum efficiency reached $85.9 \%$ at $0.042 \mathrm{~kg} / \mathrm{s}$. Copper screen matrix of $1.5 \mathrm{~m} 2$ area was studied. The matrix air collector yielded higher thermal performance with less friction losses compared to flat plate air collector.

\section{Experimental Setup 1}

The experimental set up was done by Jithinraj T, Aftab A Karim [2]. The experiment set up consists of a solar collector consisting of aluminum sheet as absorber plate, granite sheet as thermal storage medium, G.I casing and glass sheet. A drying chamber consisting of a perforated tray inside container and a blower is used. While building a solar collector at first insulation casing is made of insulating material thermocole stacked in between two GI sheets Box is about 4inch in all sides .the length and width of box is given as to accommodate $1 \mathrm{mx} 0.8 \mathrm{~m}$ aluminum sheet. The inside length of the GI box is $0.1 \mathrm{~m}$ more than the absorber sheet so that air passes from lower section of box to the upper section (two pass system). The absorber is placed inside the box; here aluminum sheet painted black is used as absorber. The dimension of the absorber is as per design $1 \times 0.8 \mathrm{~m}$. Below absorber plate granite sheet of same length and width of that of absorber sheet is placed for storing the sensible heat, the thickness of granite sheet is about $4 \mathrm{~cm}$. At the top of the absorber, glass sheet is placed which transmits maximum solar radiation falling on it. Drying cabinet consist of a small cylindrical chamber build of GI material and inside which material of high insulation properties are placed or coated so that it does not allow heat to escape out. A blower is used for continuously blow air from outside to the solar collector and 
the air is heated and the heated air is taken to drying chamber.

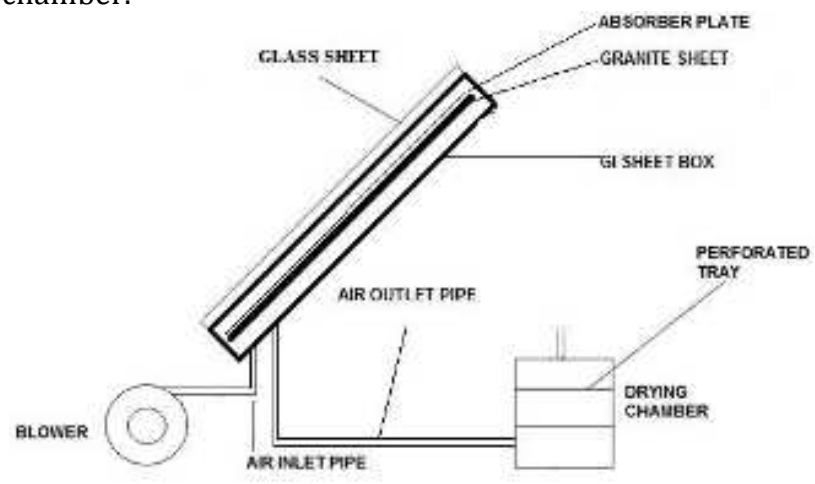

Figure: 10: Experimental layout of experimental setup

\section{J. Experimental Setup 2}

K. Rajarajeswari A. Sreekumar [4] the experiment essentially consists of matrix solar air heater, blower and a drying chamber. The area of solar air heater is $6 \mathrm{~m} 2$ with length and width of $2 \mathrm{~m}$ and $1 \mathrm{~m}$ respectively. The developed dryer consists of two parts: solar air heater and drying chamber. The peculiarity of the design is that the food is protected from direct exposure to sun. This type of indirect drying helps to retain the colour of the agricultural products. The collector consists of an outer glass cover, two layers of wire mesh followed by a bottom plate and then finally an insulation layer. The upper cover is made up of toughened glass of $4 \mathrm{~mm}$ thickness and $90 \%$ transmissivity. The bottom plate is made up of Aluminum (Al) coated with selective paint of higher absorptivity. The wire mesh absorber comprises of two parallel layers of selective coated wire mesh made up of Galvanized Iron (GI), which is separated by a spacing of $2 \mathrm{~mm}$ each other. The diameter of the wire is 1 $\mathrm{mm}$ and pitch is $3.175 \mathrm{~mm}$. Introducing wire mesh with smaller diameter and lower porosity of packing between the glass cover and the bottom plate improves the efficiency significantly, due to the turbulence provided by the packing in the airflow passage. The bottom and sides are insulated with $50 \mathrm{~mm}$ thick polyurethane foam (PUF) with a thermal conductivity of $0.16 \mathrm{~W} / \mathrm{mK}$. The inlet area of the air passage of the collector is $172 \mathrm{~cm} 2$. A centrifugal blower is provided to suck the hot air. The volume flow rate of the blower is 500 $\mathrm{m} 3 / \mathrm{h}$. The blower blows the hot air into the drying chamber where the product is loaded for drying. The dimension of the chamber is $(140 \mathrm{~cm} \times 70 \mathrm{~cm} \times 94 \mathrm{~cm})$. The loading capacity of the chamber is $30 \mathrm{~kg}$ fresh product per batch with 10 trays made up of Stainless Steel (SS). The wall of the chamber is made up of $50 \mathrm{~mm}$ PUF sandwich with SS304/GI. The collector is mounted at $12^{\circ}$ with respect to horizontal surface according to the latitude of the place.

\section{K. An Indirect Solar Dryer with the Re-Circulation of Air}

Jyoti Singh[7] In this solar dryer a duct is provided from motor to drying chamber to recirculate the hot air from motor to the chamber so the losses gets reduces and the time taken in drying the food product is less than the time taken in normal indirect dryers and from experimental results the moisture removal rate is also increased in comparison to before its approximately 80 to become $10.5 \%$ instead of $13.5 \%$.so an indirect faced solar dryer having recirculation of hot air is more suitable then others from the food preservation as well as time conservation and energy conservation also.

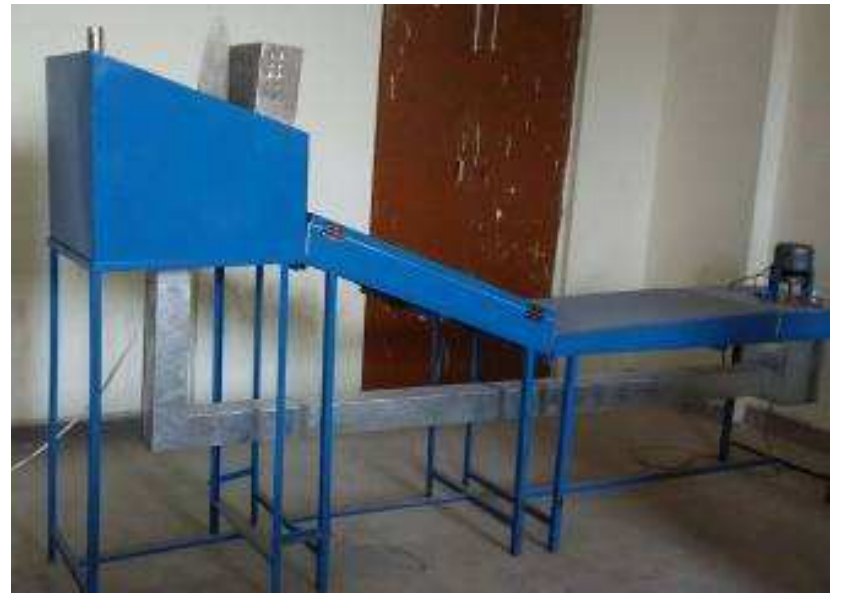

Figure12: Experimental Indirect Solar with Airflow Control

\section{Mixed Mode Solar Drying}

Jyoti Singh [7] it is combination of direct and indirect solar drying method. Product may dry with both direct exposure to solar radiation and hot air supplier on it. Air may heated in solar energy collector first then pass to the chamber where products are stored. In this process product may dry according to convective moisture loss. The System is divided into three main components: an air- heater, drying chamber, and a chimney. Air-heater through which the drying air is heated as it flows over and under an absorber plate that is heated in turn by direct absorption of incident radiation. Crop to be dried is placed in drying chamber. The moist air flows through chimney and escapes into the surrounding is partially or totally covered with the transparent material to exposure the products to solar radiation.

\section{CONCLUSION}

The present review paper concluded that lots of work for heat transfer enhancement of solar air heater by using artificial of different shapes and size is carried out with compromising with slightly more consumption of blower power. So far various correlations for heat transfer and friction factor for solar air heater duct having artificial roughness of different geometry has been investigated. This derived correlation can be used to predict the thermohydraulic as well as thermal performance of solar air heater having roughened duct.

\section{REFERENCES}

[1] Ashok Singh Yadav, Tarun Singh Samant, Lokesh Varshney, "A CFD Based Analysis of Solar Air Heater Having V-Shaped Perforated Blocks on Absorber Plate", International Research Journal of Engineering and Technology (IRJET), Volume: 02 Issue: 02, May-2015.

[2] Ajeet, Ravi, "A CFD based Heat Transfer and Fluid Flow of Solar Air Heater Provided with Combination of Circular and Square Section Transverse RIB Roughness on the Absorver Plate", IJARSE, Dec 2012.

[3] G.Padmanaban, P.K.Palani, S.Dharmalingam, K.Raja, Rathinam, "A Review on Solar dryer performance reaction on products", International Journal of ChemTech Research, CODEN (USA): IJCRGG ISSN: 0974-4290, Vol.8, No.1, 2015, page no.098-104

[4] Jithinraj T, Aftab A Karim, "Experimental Analysis on Multi Pass Flat Plate Collector Solar Air Dryer", International Journal of Emerging Engineering 
Research and Technology, ISSN 2349-4395 (Print) \& ISSN 2349-4409,Volume 2, Issue 7, October 2014, page no.1-11

[5] Manish Kumar Tated, Dinkar Prasad Singh and Sudhanshu Dogra, "Heat Transfer and Friction Factor Characteristics of Double Pass Solar Air Heater Using W-Shaped Artificial Roughness Ribs", IOSR Journal of Mechanical and Civil Engineering, (IOSR-JMCE) e-ISSN: 2278-1684, p-ISSN: 2320-334X, page no.25-30.

[6] K. Rajarajeswari, "PERFORMANCE EVALUATION OF A WIRE MESH SOLAR AIR HEATER", Voice of Research, Volume 3 Issue 3, December 2014 ISSN No. 2277-7733.

[7] Hari Om Krishna, Jihin C, Thoufeer K. V, Mohammed Dhervish P. N. M, Sujith Raj P, Shoukathali K, Muhammed saeed K, "Design and Fabrication of Multi Pass Multi Rack Solar Dryer", International Journal of Emerging Technology and Advanced Engineering,(ISSN 2250-2459),Volume 5, Issue 3, March 2015.

[8] Megha S. Sontakke, Prof. Sanjay P. Salve, "Solar Drying Technologies: A review", International Refereed Journal of Engineering and Science (IRJES) ISSN 23191821 Volume 4, Issue 4 (April 2015) ,page no.29-35.
[9] Jyoti Singh, "Review Paper of Study on Solar Dryer", Journal of Mechanical and Mechanics Engineering, Volume 1 Issue 1.

[10] Pranav C. Phadke, Pramod V .Walke and Vilayatrai M. Kriplan, "A REVIEW ON INDIRECT SOLAR DRYERS", Journal of Engineering and Applied Sciences, ISSN 1819-6608 ARPN,VOL. 10, NO. 8, MAY 2015,

[11] Srivastava A.K, Shukla S.K, Singh, "Modelling and Evaluation of Thermal Diffusivity and Activation Energy of Potato slices in Forced Convection Multi Tray Solar Dryer", American Journal of Food Science and Technology, 2015, Vol. 3, page no.27-32

[12] Raj Kumar , Ranchan Chauhan , Muneesh Sethi, "Thermal Analysis and Recent Developments in Artificially Roughened Solar air Heater", Journal of Material Science and Mechanical Engineering (JMSME), Print ISSN: 2393-9095; Online ISSN: 2393-9109, Volume 1, Number 1; October, 2014, page no.7-10.

[13] Santosh Vyas and Dr. Sunil Punjabi, "THERMAL PERFORMANCE TESTING OF A FLAT PLATE SOLAR AIR HEATER USING OPTICAL MEASUREMENT TECHNIQUE", International Journal of Recent advances in Mechanical Engineering (IJMECH), Vol.3, No.4, November 2014. 